\title{
BODIPY-based dye for no-wash live-cell staining and imaging
}

\begin{abstract}
Alexey A. Pakhomov¹, Igor E. Deyev¹, Natalia M. Ratnikova', Stepan P. Chumakov ${ }^{1}$, Veronika B. Mironiuk ${ }^{1,2}$, Yuriy N. Kononevich², Aziz M. Muzafarov ${ }^{2,3}$, and Vladimir I. Martynov ${ }^{1}$ ${ }^{1}$ M.M. Shemyakin and Yu.A. Ovchinnikov Institute of Bioorganic Chemistry, Moscow, Russian Federation, ${ }^{2}$ A. N. Nesmeyanov Institute of Organoelement Compounds, Moscow, Russian Federation, and ${ }^{3}$ N.S. Enikolopov Institute of Synthetic Polymeric Materials, Moscow, Russian Federation
\end{abstract}

BioTechniques 63:77-80 (August 2017) doi 10.2144/000114577

Keywords: fluorophore; aggregation-caused quenching; BODIPY; live-cell imaging, flow cytometry; fluorescence microscopy

Supplementary material for this article is available at www.BioTechniques.com/article/114577.

In nonpolar solvents, hydrophobic organic fluorophores often show bright fluorescence, whereas in polar media, they usually suffer from aggregation-caused quenching (ACQ). Here, we harnessed this solvatochromic behavior of a 1,3,5,7-tetramethyl-BODIPY derivative for cell staining and applied it to live-cell imaging and flow cytometry. As opposed to commercially available dyes, this BODIPY derivative showed excellent contrast immediately after staining and did not require any wash-off.

A variety of small-molecule synthetic fluorophores have been developed for live-cell imaging in recent years. These fluorescent molecules can either be introduced nonspecifically to uniformly stain the cells or be targeted to specific cell populations, cell organelles, or selectively label a protein of interest $(1,2)$. A significant number of studies have been devoted to boron dipyrromethene (BODIPY)-based fluorophores, since these dyes are neutrally charged and exhibit excellent photostability, high quantum yield, and sharp absorption and emission bands $(3,4)$. BODIPY derivatives have been used in the development of fluorescent sensors and indicators for $\mathrm{pH}$, metal ions, reactive oxygen species (ROS), nitric oxide (NO), redox potential, etc. (5-10).
Due to high lipophilicity, some BODIPY derivatives tend to adhere to and penetrate the cell membrane and accumulate in subcellular membranes (11). Generally, the lipophilicity of BODIPY derivatives is considered a drawback that limits their bioanalytical applications. In a polar medium, such as the cytosol or extracellular space, these fluorophores tend to aggregate and are prone to aggregation-caused quenching (ACQ) (12). Here, we show how to take advantage of these seemingly unfavorable properties of BODIPY dyes to improve live-cell staining and imaging protocols.

Previously we reported the synthesis of a tetra-BODIPY-substituted siloxane fluorescent dye comprising four 1,3,5,7-tetramethyl-BODIPYs (TMBs) conjugated to a cyclotetrasiloxane core (tetra-TMB) (Supplementary Figure S1, Compound 1) (13). Preliminary studies indicated that tetra-TMB exhibits a significant quantum yield drop in polar solvents. This solvatochromism was shown to be due to interfluorophore interactions of the lipophilic TMB groups concentrated within the star-shaped molecule (Supplementary Table S1) (13). Here, we tested several previously synthesized 1,3,5,7-tetramethyl-BODIPY derivatives (monoTMBs) $(13,14)$ for their ability to exhibit a similar solvatochromic behavior and report the use of mono-TMB Derivative 2 (mono-TMB 2) (Supplementary Figure $\mathrm{S} 1$, Compound 2; Figure 1A, insert) as a wash-free live-cell-labeling dye. In the polar extracellular space, the fluorescence of this dye is turned off due to $A C Q$, whereas when absorbed by the membrane lipid bilayer, the dye brightly fluoresces.

In contrast to tetra-TMB 1, mono-TMB 2 did not display considerable solvatochromism in organic solvents and was not subject to self-quenching. In polar and nonpolar organic solvents, mono-TMB 2 showed characteristic sharp absorption and emission bands, with maxima at $\sim 500 \mathrm{~nm}$ and $\sim 510 \mathrm{~nm}$, respectively, and a quantum yield of 90\% (Figure 1A; Supplementary Table S1). At the same time, mono-TMB 2 proved poorly soluble in water. In order to define its optical characteristics, the dye was dissolved in tetrahydrofuran (THF) at a concentration of $10^{-2} \mathrm{M}$ and then diluted in water to $10^{-4}-10^{-6} \mathrm{M}$. Dilution was accompanied by partial precipitation of the dye aggregates. The residual water-soluble mono-TMB 2 showed a reddish color; it was weakly fluorescent under UV light and showed broadened absorption and emission bands consistent with interfluorophore interactions (12).

To compare the properties of the dye in aqueous and nonpolar organic solvents, a $10^{-2} \mathrm{M}$ stock solution of mono-TMB 2 in THF was diluted 100-fold with mineral oil (high-boiling

\section{METHOD SUMMARY}

Here, we describe a BODIPY-based dye that switches on fluorescence when absorbed by the cell membrane and turns off fluorescence in aqueous medium. The dye exhibits excellent contrast immediately after cell staining and does not require any wash-off for fluorescence cell imaging and flow cytometric experiments. 
petroleum fraction) (Figure 1B, Test Tube 1) or water (Figure 1B, Test Tube 2). Mono-TMB 2 showed solvatochromic behavior similar to that of tetra-TMB 1 , with bright fluorescence in oil and quite dim fluorescence in water. After addition of mineral oil to the mono-TMB 2 in aqueous solution and subsequent phase separation, we observed the lipophilic dye transition to the upper oil phase with a concomitant increase in fluorescence (Figure 1B, Test Tube 3). Consequently, we considered this system as a model for the lipophilic cell membrane surrounded by aqueous medium. According to our data, addition of mono-TMB 2 in aqueous solution to live cells would selectively stain the cells shortly after the dye had been absorbed by the cell membrane. A carboxylic derivative of mono-TMB (Supplementary Figure S1, Compound 3) proved to be unsuitable for cell membrane labeling, since it more readily dissolved in water (Supplementary Figure S2). These experiments indicated that a balance between lipophilicity, water solubility, and $A C Q$ in various media should be taken into account when using TMB derivatives for cell labeling.

Next, we stained live mammalian cells with tetra-TMB 1 and mono-TMB 2 (Figure 1C; Supplementary Figures S3 and S4, top two panels). Fluorescence microscopic visualization of cells showed high fluorescence signal contrast between the extracellular space and the cells. When compared with mono-TMB 2, tetra-TMB 1 was less promising for cell labeling due to its lower quantum yield ( 60\%) in nonpolar media. In contrast, mono-TMB 2 displayed a $90 \%$ fluorescence quantum yield in organic solvents, whereas in aqueous solution fluorescence was quenched due to $A C Q$. Although both derivatives may be used for cell labeling, mono-TMB 2 displayed superior contrast. Given the facile synthesis of mono-TMB 2, that compound has the highest potential for bioanalytical applications.

Upon addition of the aqueous solution containing mono-TMB 2 to a final concentration of $10^{-6} \mathrm{M}$, the cells were immediately stained with a high contrast that enabled instant visualization using a fluorescence microscope. Staining of the outer plasma
A

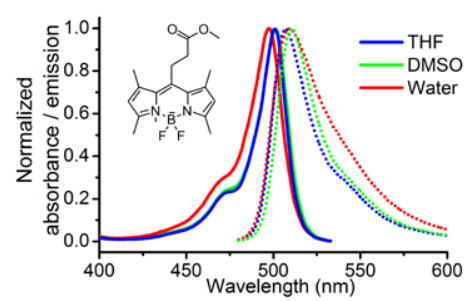

B

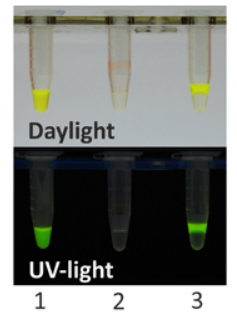

C
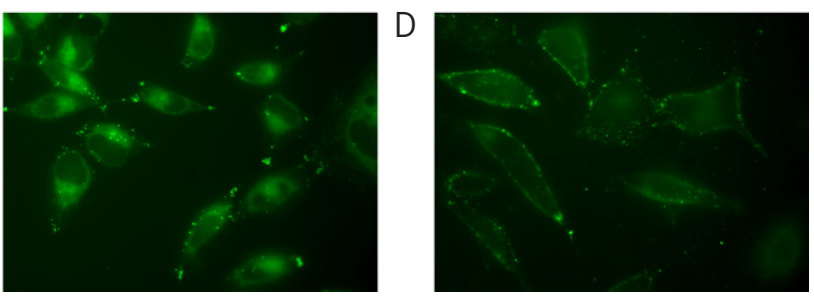

Figure 1. Optical properties of mono-TMB Derivative 2 (monoTMB 2) and live-cell staining. (A) Absorption (solid lines) and fluorescence emission (dotted lines) spectra of mono-TMB 2 in various solvents. (B) Fluorescence of mono-TMB 2 in mineral oil (Test Tube 1), water (Test Tube 2) and in the water-mineral oil mixture (Test Tube 3). (C) Fluorescence microscopy image of HeLa cells stained with mono-TMB 2. (D) HeLa cells stained with Vybrant DiO dye.

membrane and subcellular membrane structures was observed (Figure 1C; Supplementary Figure S4, top two panels). As was pointed out above, some BODIPY derivatives show a similar propensity to adhere to and penetrate the cell membrane and to accumulate in subcellular membrane structures (11). In contrast, the commercial lipophilic carbocyanine dye Vybrant DiO (Molecular Probes/Thermo Fisher Scientific, Eugene, OR) predominantly stained the plasma membrane (15). This dye showed a contrast similar to that of

mono-TMB 2 upon staining (Figure 1D; Supplementary Figure S4, bottom two panels). However, to attain the best contrast, it took $10 \mathrm{~min}$ at $37^{\circ} \mathrm{C}$ to stain cells with $\mathrm{DiO}$, and there was no staining at room temperature. To eliminate the unbound DiO dye, there was need for a stepwise washing off procedure, which took $10 \mathrm{~min}$ at $37^{\circ} \mathrm{C}$ for every washing step. In contrast, mono-TMB 2 stained the cells within seconds at room temperature.

Mono-TMB 2 was further tested for possible use in fluorescence
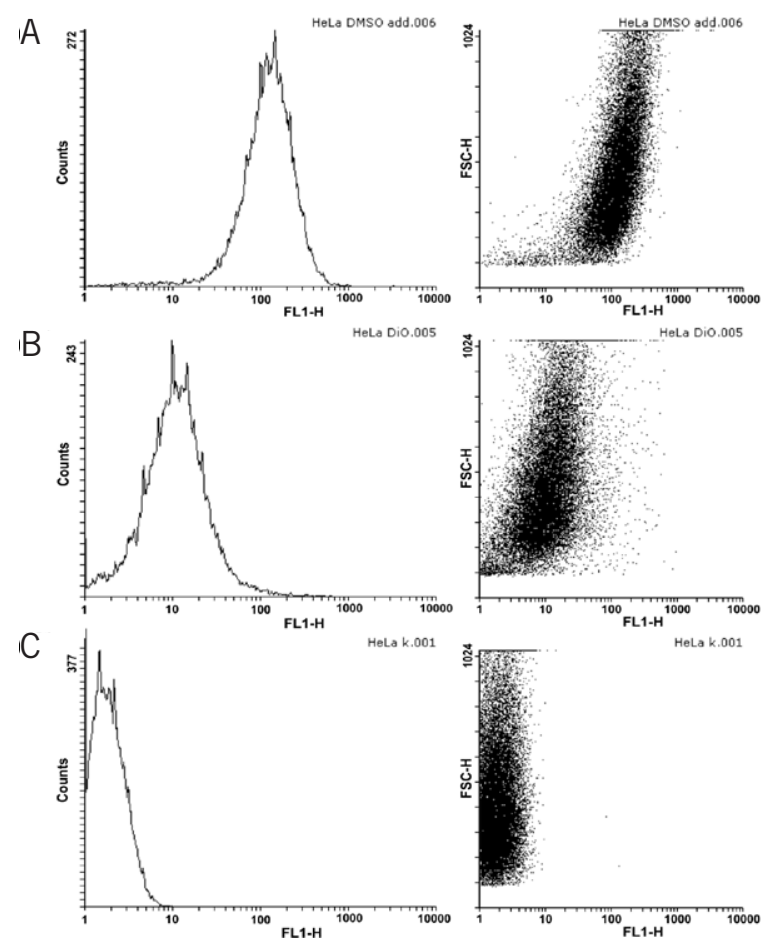
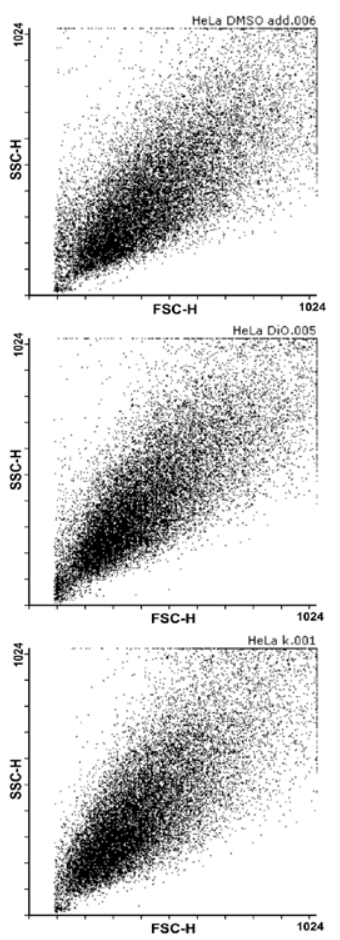

Figure 2. Fluorescence flow cytometric analysis. HeLa cells stained with mono-TMB Derivative 2 (mono-TMB 2) (A), Molecular Probes Vybrant DiO dye (B), or unstained (C). FL1: fluorescence emission 530/30 nm; FCS: forward scattering; SSC: side scattering. 
flow cytometry. Labeled cells were detached using trypsin, washed, and resuspended in PBS. Cells labeled with DiO showed an increase in the green fluorescence signal of the stained cell population (Figure 2B) compared with unstained cells (Figure 2C). Unexpectedly, we could not distinguish mono-TMB 2-stained cells from the unstained ones (data not shown). Fluorescence microscopy revealed that mono-TMB 2 had been removed from the cells after the washing procedure, suggesting a reversible dye coupling. Next, we added a solution of mono-TMB 2 to a final concentration of $10^{-6} \mathrm{M}$ directly to the cell suspension after trypsin treatment and the washing procedure. Immediately after the dye was added, flow cytometric analysis showed extensive fluorescent staining, and there was no need for washing off the unbound dye (Figure 2A). Thus, mono-TMB 2 can be used as a wash-free reversible cell-staining dye. These results imply that the dye is in an equilibrium partitioning between water and the preferred cell membrane lipid phase. This equilibrium partitioning allows more rapid uniform labeling of cells. Moreover, the cells labeled with mono-TMB 2 can be destained and stained again with another dye, followed by fluorescence microscope visualization in the same spectral range.

Here, we report the development and successful use of a new cellstaining dye based on a BODIPY derivative with a sufficiently simple structure and facile synthesis. The dye displays pronounced solvatochromic behavior that enables bright and uniform cell staining. Compared with previously reported dyes, mono-TMB 2 can be used for instant fluorescence cell visualization, for example, in cell proliferation or motility assays. Mono-TMB 2 shows excellent signal contrast without the need for the preliminary washing off of the unbound dye, which is advantageous in experiments where the washing off procedure is undesirable. Owing to Mono-TMB 2's reversible coupling to the membrane, the cells can be destained and used again for subsequent staining and visualization in the same green channel of a fluorescence microscope.

\section{Author contributions}

A.A.P., I.E.D., A.M.M., and V.I.M. developed the research strategy, designed experiments, and analyzed the data. A.A.P., I.E.D., N.M.R., S.P.C., V.B.M., and Y.N.K. performed the experiments. A.A.P. and V.I.M. wrote the manuscript.

\section{Acknowledgments}

This work was in part supported by the Russian Science Foundation (project 14-1301478) and by the Russian Foundation for Basic Research (project 15-34-70033).

\section{Competing interests}

The authors declare no competing interests.

\section{References}

1. Zhu, H., J. Fan, J. Du, and X. Peng. 2016. Fluorescent probes for sensing and imaging within specific cellular organelles. Acc. Chem. Res. 49:2115-2126.

2. Martynov, V.I., A.A. Pakhomov, N.V. Popova, I.E. Deyev, and A.G. Petrenko. 2016. Synthetic fluorophores for visualizing biomolecules in living systems. Acta Naturae. 8:33-46.

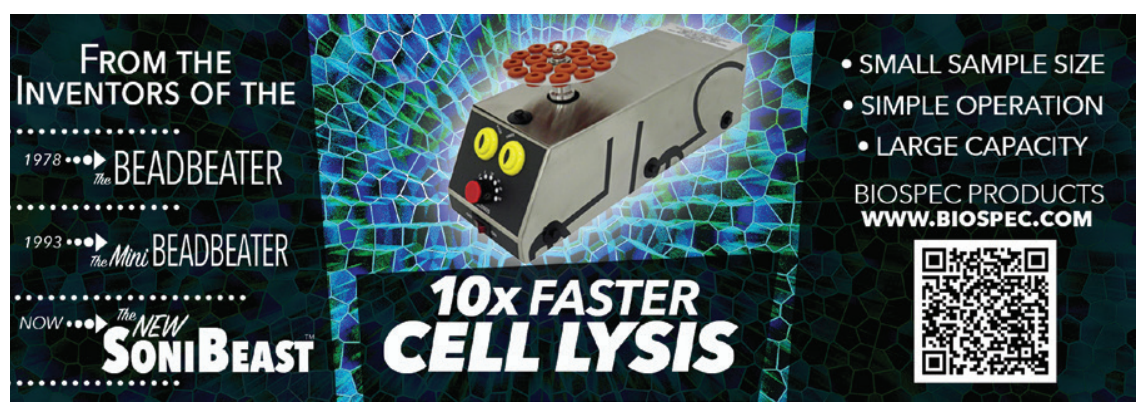

3. Kowada, T., H. Maeda, and K. Kikuchi. 2015. BODIPY-based probes for the fluorescence imaging of biomolecules in living cells. Chem. Soc. Rev. 44:4953-4972.

4. Loudet, A. and K. Burgess. 2007. BODIPY dyes and their derivatives: Syntheses and spectroscopic properties. Chem. Rev. 107:4891-4932.

5. Boens, N., V. Leen, and W. Dehaen. 2012. Fluorescent indicators based on BODIPY. Chem. Soc. Rev. 41:1130-1172.

6. Li, Z.S., L.J. Li, T.T. Sun, L.M. Liu, and Z.G. Xie. 2016. Benzimidazole-BODIPY as optical and fluorometric $\mathrm{pH}$ sensor. Dyes Pigm. 128:165-169.

7. Qin, W.W., W. Dou, V. Leen, M. Van der Auweraer, and N. Boens. 2016. A ratiometric, fluorescent BODIPY-based probe for transition and heavy metal ions. RSC Advances 6:78067816

8. Zhang, H.X., J.B. Chen, X.F. Guo, H. Wang, and H.S. Zhang. 2013. Highly sensitive determination of nitric oxide in biologic samples by a near-infrared BODIPY-based fluorescent probe coupled with high-performance liquid chromatography. Talanta 116:335-342.

9. Yamada, Y., Y. Tomiyama, A. Morita, M. Ikekita, and S. Aoki. 2008. BODIPY-based fluorescent redox potential sensors that utilize reversible redox properties of flavin. ChemBioChem 9:853-856.

10. Rubinstein, N., P. Liu, E.W. Miller, and R. Weinstain. 2015. meso-Methylhydroxy BODIPY: a scaffold for photo-labile protecting groups. Chem. Commun. (Camb.) 51:63696372.

11. Jiao, L., C. Yu, T. Uppal, M. Liu, Y. Li, Y. Zhou, E. Hao, X. Hu, and M.G.H. Vicente. 2010. Long wavelength red fluorescent dyes from 3,5-diiodo-BODIPYs. Org. Biomol. Chem. 8:2517-2519.

12. Huang, M.N., R.N. Yu, K. Xu, S.X. Ye, S. Kuang, X.H. Zhu, and Y.Q. Wan. 2016. An arch-bridge-type fluorophore for bridging the gap between aggregation-caused quenching (ACQ) and aggregation-induced emission (AIE). Chem. Sci. (Camb.) 7:4485-4491.

13. Pakhomov, A.A., Y.N. Kononevich, M.V. Stukalova, E.A. Svidchenko, N.M. Surin, G.V. Cherkaev, O.I. Shchegolikhina, V.I. Martynov, and A.M. Muzafarov. 2016. Synthesis and photophysical properties of a new BODIPY-based siloxane dye. Tetrahedron Lett. 57:979-982.

14. Pakhomov, A.A., Y.N. Kononevich, A.A. Korlyukov, V.I. Martynov, and A.M. Muzafarov. 2016. Synthesis, crystal structure and optical properties of a new meso-acrylate BODIPY dye. Mendeleev Commun. 26:196-198.

15. Johnson, I. and M.T.Z. Spence, eds. 2010. Molecular Probes Handbook: A Guide to Fluorescent Probes and Labeling Technologies, 11th Edition, Life Technologies, Eugene, OR.

Received 16 March 2017; accepted 05 June 2017.

Address correspondence to Alexey A. Pakhomov, Shemyakin and Ovchinnikov Institute of Bioorganic Chemistry, Russian Academy of Sciences, Miklukho-Maklaya 16/10, 117997 Moscow, Russian Federation. E-mail: alpah@mail.ru 